\title{
Effects of different parameters on transient heat transfer surrounding energy piles in unsaturated soils
}

\author{
Davood Yazdani Cherati ${ }^{1}$, Omid Ghasemi-Fare ${ }^{1, *}$, and Hussein Hashemi Senejani ${ }^{2}$ \\ ${ }^{1}$ Department of Civil and Environmental Engineering, University of Louisville, USA \\ ${ }^{2}$ Department of Civil Engineering, Sharif University, Tehran, Iran
}

\begin{abstract}
Energy piles are one of the best candidates to harvest shallow geothermal energy. Continuing harvesting and rejecting thermal energy from/to the ground may permanently change the soil temperature and consequently, may lead to different soil resistance parameters. Therefore, careful prediction of soil temperature variation close to energy geostructures is needed. This paper investigates the various parameters of heat source and soil media on transient heat transport in unsaturated soils. Green's function and the method of separation of variables are employed to analytically analyze the transient heat transfer in the vicinity of energy piles. Results indicate that although higher heat flux increases the soil temperature surrounding a heat source, it has negligible effect on thermal influence zone where the soil temperature varies. In contrast, higher thermal diffusivity results in lower soil temperature close to a heat source while it increases the thermal influence zone.
\end{abstract}

\section{Introduction}

Recently energy piles have been employed around the world to partially heat and cool buildings to reduce the use of fossil fuels. [1]. Application of energy geostructures may significantly change the soil temperature. Soil properties (e.g., water content, and strength) is dependent on soil temperature. However, soil temperature can be different at different time steps. Therefore, in order to accurately predict the performance of energy geostructures, and the energy efficiency of energy piles, heat transfer in the vicinity of heat sources (e.g., energy piles) should be analyzed in a transient condition. Several researches have been performed using numerical, experimental and analytical models in literature to analyze the heat transfer in the soil media and predict the soil temperature close to a heat source. Among those, analytical models provide the exact solution for simplified conditions which can be used to estimate the performance and provide the tools to validate complicated numerical models. Solving heat transfer mechanism in unsaturated soil can be complicated. However, in order to make the analytical solution possible, several simplification assumptions can be considered. Heat convection flow can be neglected in hydrostatic condition to reduce the nonlinearity of the model. Note, the thermally induced pore fluid flow under hydrostatic condition has limited effect on soil temperature response [2]. In addition, the evaporation term has negligible effect on temperature profile of the soil [3]. Therefore, it is possible to consider heat conduction as the sole heat transfer mechanism in soil.
Several attempts have been made in the literature to analytically solve the heat transfer close to energy piles in soil media considering the above-mentioned assumption. Eskilson and Claesson proposed a model to predict the performance of the heat pump systems including single and multiple boreholes in porous media [4]. Zeng et al. proposed analytical models for the heat transfer from ground heat exchangers to the surrounding soil $[5,6]$. Lamarche and Beauchamp developed an analytical approach to predict the short time response of geothermal vertical boreholes [7]. To predict the energy efficiency of the energy piles, Ghasemi-Fare and Basu presented a semi-analytical model considering variable inlet temperature $[8,9]$. Later, they predicted the soil temperature response for both short term and long term close to the energy piles [10]. However, most of the existing models have not studied the different shapes and locations of the source terms and soil parameters on heat transfer in soil media. In addition, only limited research is available in literature which proposed the heat and moisture transport in unsaturated media using analytical solution [11]. In this study same approach presented by Cherati and Ghasemi-Fare [11] is used to predict the effects of different parameters on transient heat and water transport in unsaturated soil media.

\section{Explanation of the employed model}

In order to analyze the heat transfer mechanism in unsaturated soils, heat equation should be solved in this study. Note, hydrostatic condition is assumed and heat

* Corresponding author: Omid.ghasemifare@1ouisville.edu 
convection in the ground is ignored in this study for simplicity. A heat source term in heat conduction equation represents an energy pile and the method of separation of variables and Green's function are used to solve the equations analytically. The approach used in this study was validated in a previous study by Cherati and Ghasemi-Fare. Figure 1 shows the schematic geometry of the model and table 1 presents the assumptions and procedures considered in this study [11].

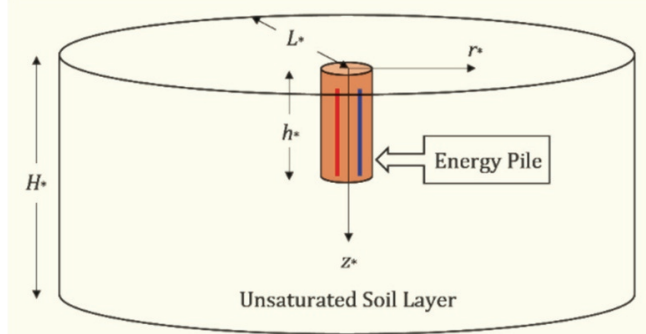

Fig. 1. Schematic geometry of the model including Energy pile in a soil layer presented by Cherati and Ghasemi-Fare [11]

Table 1. Assumptions and procedures

\begin{tabular}{|c|l|}
\hline $\begin{array}{c}\text { Assumption } \\
\text { process }\end{array}$ & $\begin{array}{l}\text { Eydrostatic condition is assumed and } \\
\text { therefore the heat convection flow is } \\
\text { neglected. } \\
\text { In order to analytically solve the } \\
\text { equation, the nonlinear evaporation term } \\
\text { is neglected. }\end{array}$ \\
\hline $\begin{array}{c}\text { Effects of the } \\
\text { Source term }\end{array}$ & $\begin{array}{l}\text { A heat generation term is considered to } \\
\text { represent the heat flux from the energy } \\
\text { piles. }\end{array}$ \\
\hline $\begin{array}{c}\text { Initial and } \\
\text { boundary } \\
\text { conditions }\end{array}$ & $\begin{array}{l}\text { Constant temperature in all boundaries } \\
\text { equal to initial condition is considered. }\end{array}$ \\
\hline Coordinate system & $\begin{array}{l}\text { A 2D axisymmetric cylindrical } \\
\text { coordinate system is used }\end{array}$ \\
\hline Solution procedure & $\begin{array}{l}\text { The separation of variables is used to } \\
\text { reduce the PDEs to ODEs and then } \\
\text { Green's function is employed to solve the } \\
\text { ODEs. }\end{array}$ \\
\hline Characteristics of \\
the model & $\begin{array}{l}\text { The model presents an exact closed form } \\
\text { solution for heat and heat induced } \\
\text { moisture flow in unsaturated soil. } \\
\text { Compared to other available analytical } \\
\text { solution, the current model can predict } \\
\text { the moisture behavior in unsaturated } \\
\text { soils in addition to heat transfer. } \\
\text { The employed model can consider } \\
\text { different parameters for unsaturated soil } \\
\text { and can be updated considering variable } \\
\text { boundary and initial conditions at the } \\
\text { final stage. } \\
\text { The defined heat source term can be used } \\
\text { to model various conditions in practice. } \\
\text { It can be used to model constant sources } \\
\text { and sources which attenuates with } \\
\text { different rates over time. }\end{array}$ \\
\hline
\end{tabular}

The dimensions (length and diameter) and the location of the heat source are defined by the upper and lower limits of the integral of the Green's function. For example, if the source term (energy pile) is placed from depth of $5 \mathrm{~m}$ to $15 \mathrm{~m}$, the upper and lower limits for the integral related to depth will be 15 and 5, respectively. A hollow cylinder model can also be considered using this approach if the upper and lower limits of the integral concerned with the radius change from outer to inner diameters values, respectively.

The heat conduction equation with generation term can be expressed as:

$$
\frac{\partial T_{*}}{\partial t_{*}}=\frac{\lambda}{\rho c} \nabla^{2} T_{*}+Q_{*}
$$

where $T_{*}$ is temperature, $t_{*}$ is time, $\lambda$ is thermal conductivity, $\rho c$ is volumetric heat capacity of the porous medium, * indicates dimensional variables, and $Q_{*}$ is a heat source which can be predicted using Eq. (2).

$$
Q_{*}=C_{*} e^{v t}
$$

In which $C_{*}\left[\frac{{ }^{\circ} \mathrm{C}}{s}\right]$ and $v$ are constant and $v$ is a non-positive number, $t$ is the dimensionless time which will be defined later in Eq (4). When a negative value is used for $v$, heat generation term attenuates and reaches to zero eventually.

Initial and boundary conditions mentioned in the Table 1. can be presented as:

$$
\begin{aligned}
& T_{*}\left(r_{*}, Z_{*}, 0\right)=T_{*}\left(r_{*}, H_{*}, t_{*}\right)=T_{*}\left(L_{*}, Z_{*}, t_{*}\right)= \\
& T_{*}\left(r_{*}, 0, t_{*}\right)=T_{0}
\end{aligned}
$$

In order to solve the equations, dimensionless parameters are introduced and replaced in Eqs. 1 and 2.

$$
\begin{aligned}
& r=\frac{r_{*}}{a_{*}}, Z=\frac{z_{*}}{a_{*}}, H=\frac{H_{*}}{a_{*}}, L=\frac{L_{*}}{a_{*}}, h=\frac{h_{*}}{a_{*}}, t= \\
& \frac{D_{\theta} t_{*}}{a_{*}{ }^{2}}, T=\frac{T_{*}-T_{0}}{T_{0}}, \chi=\frac{\frac{\lambda}{\rho c}}{D_{\theta}}, C=\frac{C_{*} a_{*}{ }^{2}}{D_{\theta} T_{0}}
\end{aligned}
$$

where $D_{\theta}$ is isothermal moisture diffusivity, $a_{*}$, and $h_{*}$ are radius and height of the energy pile, and $L_{*}$ and $H_{*}$ are radius and height of the domain, respectively. Using new dimensionless variables, dimensionless energy equation is obtained as presented in Eq. (5).

$$
\frac{1}{\chi} \frac{\partial T}{\partial t}=\nabla^{2} T+Q \quad Q=C e^{v t}
$$

New dimensionless initial and boundary conditions are expressed as:

$$
T(r, z, 0)=T(r, H, t)=T(L, z, t)=
$$




$$
T(r, 0, t)=0
$$

The analytical solution to predict soil temperature is optioned based on Green's function.

$$
\begin{aligned}
& T(r, z, t)= \\
& \chi \int_{\tau}^{t} \mathrm{~d} \tau \int_{A} r G_{T}(r, z, t \mid r, z, \tau) \operatorname{gen}(r, z, \tau) \mathrm{d} A
\end{aligned}
$$

\section{Parametric study}

In this study, three different conditions are considered to investigate the effect of different parameters on transient heat transport in soil media. For all three conditions with different pile, and soil properties, PDEs are solved for two different cases considering constant and variable heat sources. Case 1 has a constant heat source while the heat source in case 2 attenuates over time. Generation term is $v=0$ for case 1 and $v=-0.5$ for case 2 . All other input parameters are presented in Table 2. All computations and figures are presented based on dimensionless variables presented in Eq. (3).

Table 2. Parameters used in this study

\begin{tabular}{llllll}
\hline$L *$ & $H$ & $C$ & $T_{0}(C)$ & $\frac{\lambda}{\rho C}\left(m^{2} / s\right)$ & $D_{\theta}\left(m^{2} / s\right)$ \\
$(m)$ & $*(m)$ & $*(C / s)$ & & & \\
\hline 50 & 50 & 5 & 20 & $8 \times 10^{-7}$ & $10^{-6}$ \\
& & $\times 10^{-5}$ & & &
\end{tabular}

Please note, although the developed model can predict both soil temperature and moisture content (heat and water flow) in soil media, in this study only the changes in soil temperature is investigated.

\section{Model 1: Effects of shape and location of the energy pile}

In this condition three heat sources are considered separately with different shape and locations. The first model is a solid cylinder with non-dimension radius of 1 which is embedded from depth 0 to 15 . The second model is a hollow cylinder source model with inner radius of 0.5 and outer radius of 1 which is placed from depth 20 to 35 ; and the third one is a disc source model with inner radius of 5 and outer radius of 10 which is embedded from depth 20 to 21 . All other parameters are the same as the Table 2. Results are computed and plotted for both constant and variable heat flux. It is expected that temperature increment close to the heat source should be higher compared to farther points. This trend can be seen in Figures 2 and 3. In addition, the figures indicate that the time corresponding to the peak point depends only on radius, and does not change over depth. By comparing the changes of temperature in depth and radius, it can be seen that the changes of temperature radially are more than temperature variations along the depth in both cases. 


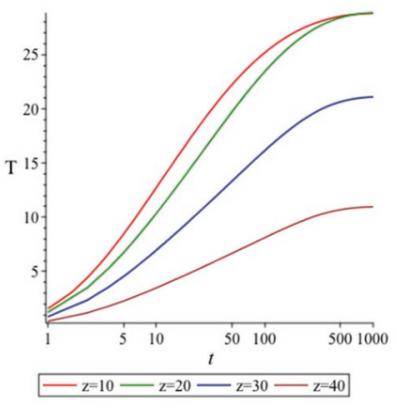

(a) source 1 of case 1

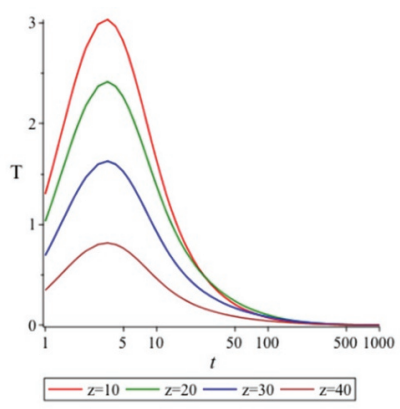

(d) source 1 of case 2

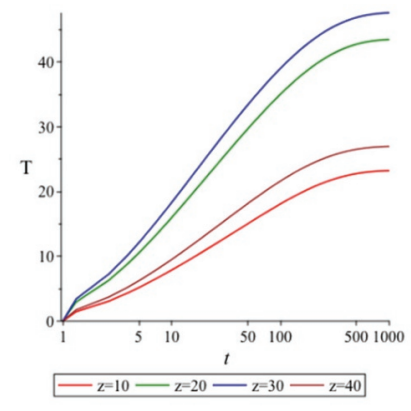

(b) source 2 of case 1

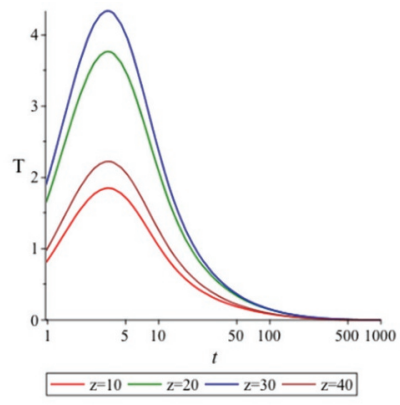

(e) source 2 of case 2

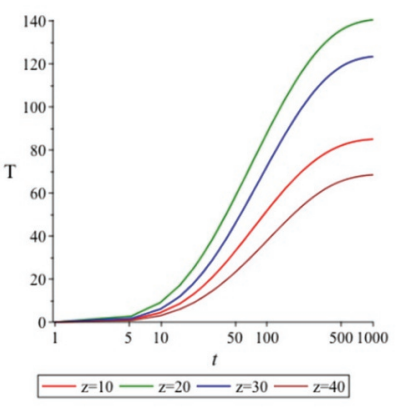

(c) source 3 of case 1

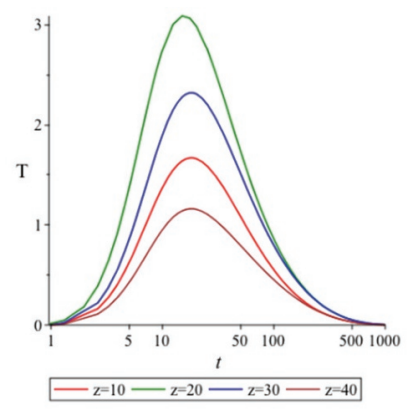

(f) source 3 of case 1

Fig. 2. Variation of temperature with time at $\mathrm{r}=2$ for both cases

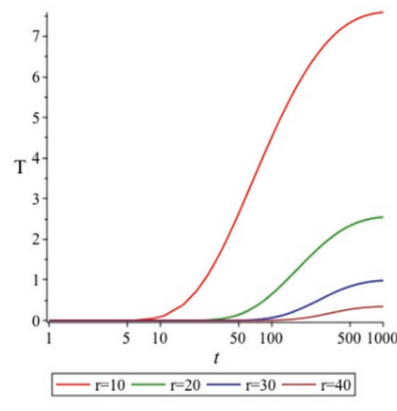

(a) source 1 of case 1

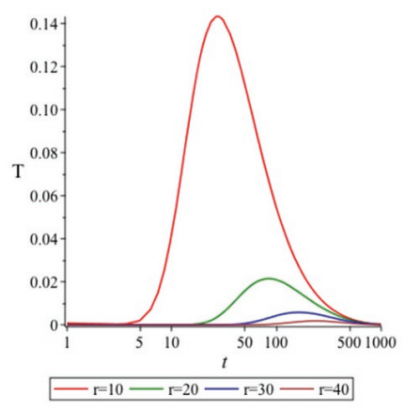

(d) source 1 of case 2

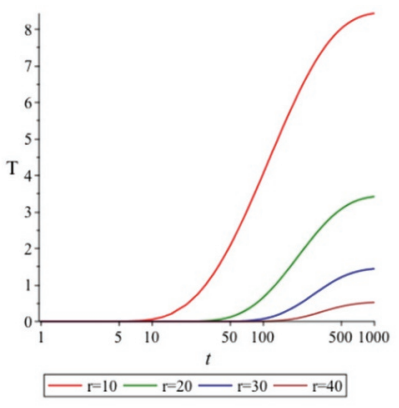

(b) source 2 of case 1

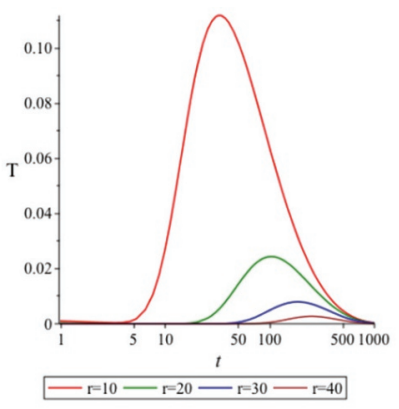

(e) source 2 of case 2

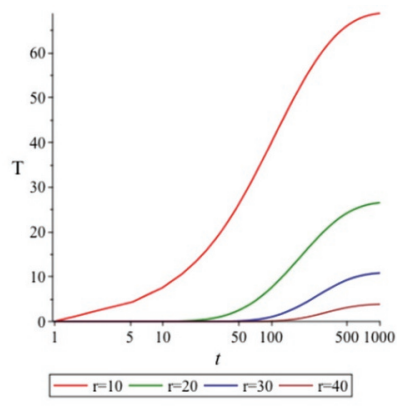

(c) source 3 of case 1

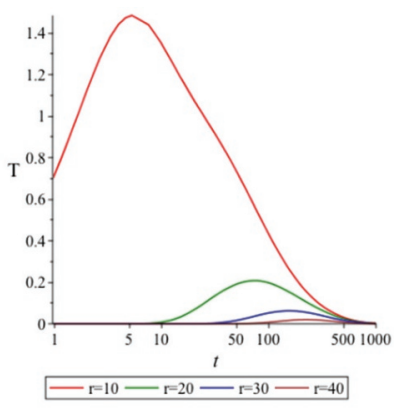

(f) source 3 of case 2

Fig. 3. Variation of temperature with time at $\mathrm{z}=10$ for two cases 


\section{Model 2: Effects of the parameter of $v$}

This example investigates the effects of parameter of $v$ on transient heat transfer in soil media for the first heat source (A solid cylinder with radius of 1 in depth of 0 to 15). Other parameters are based on Table 2.

According to Figure 4, utilizing lower $v$ value reduces both temperature and the required time to reach to the maximum temperature. However, the effects of heat flux on thermal influence zone is negligible.

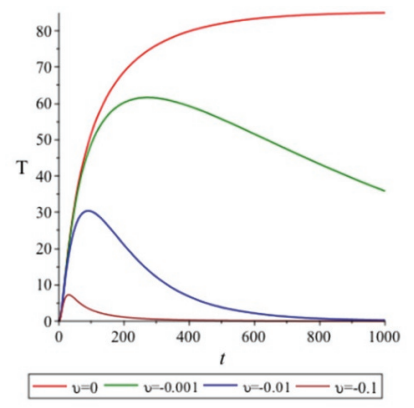

(a) at $\mathrm{r}=2$ and $\mathrm{z}=10$

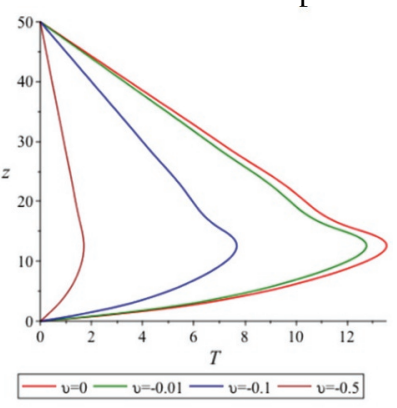

(b) at $\mathrm{r}=2$ and $\mathrm{t}=10$

\section{Model 3: Effects of the soil parameters}

In this model the effects of soil parameters are studied using the parameter of $\chi$ which depends on thermal conductivity and heat capacity of the soil. Other parameters are based on Table 2. According to Figure 5 , by increasing the parameter of $\chi$, initially soil temperature decreases and then increases gradually. Furthermore, the time corresponding to maximum temperature values increases. Increasing the parameter of $\chi$ results in higher temperature close to a heat source in radial distances and lower temperature in farther points.

Fig. 4. Variation of temperature at different values of $v$

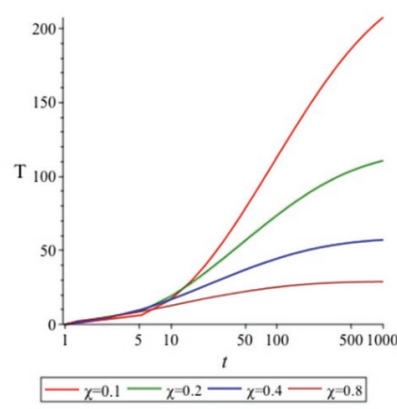

(a) at $\mathrm{r}=2$ and $\mathrm{z}=10$ for case 1

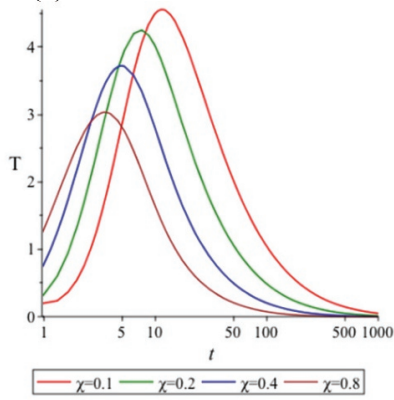

(d) at $\mathrm{r}=2$ and $\mathrm{z}=10$ for case 2

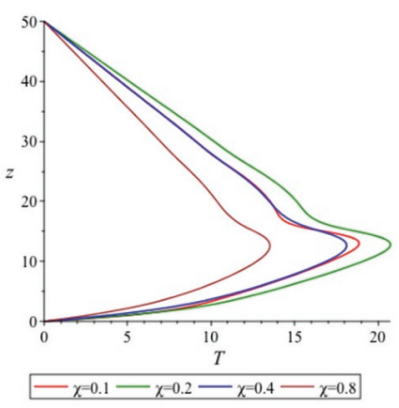

(b) at $\mathrm{r}=2$ and $\mathrm{t}=10$ for case 1

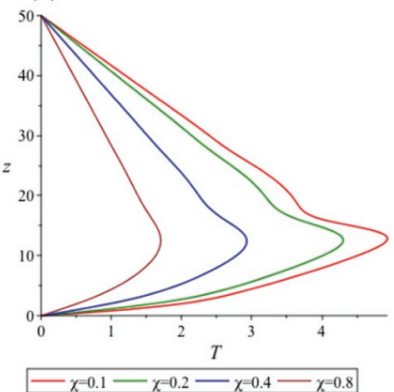

(e) at $\mathrm{r}=2$ and $\mathrm{t}=10$ for case 2

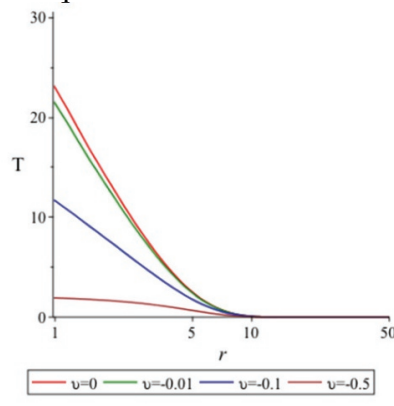

(c) at $\mathrm{z}=10$ and $\mathrm{t}=10$

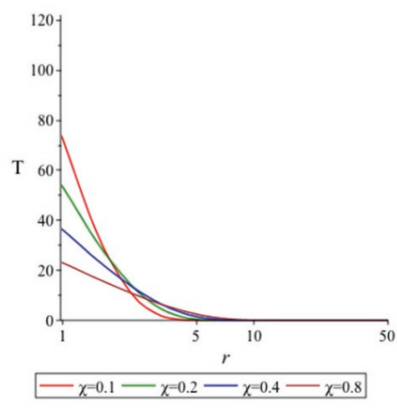

(c) at $\mathrm{z}=10$ and $\mathrm{t}=10$ for case 1

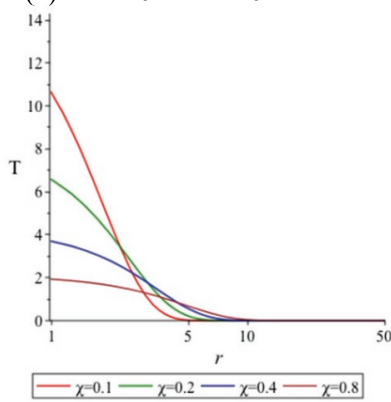

(f) at $\mathrm{z}=10$ and $\mathrm{t}=10$ for case 2

Fig. 5. Variation of temperature at different values of $\chi$ 


\section{Results and discussion}

By comparing the changes of temperature in depth and radius, it can be seen that the changes of temperature radially are more than temperature variation along the depth in both cases and different locations of the pile.

Reducing the $v$ (heat flux) results in lower soil temperature and shorten the time required to reach to the maximum soil temperature. However, the heat flux does not have significant effect on the thermal influence zone in which soil temperature changes.

Increasing the soil thermal diffusivity (the parameter of $\chi$ ) in both cases temperature soil temperature is reducing in the vicinity of a heat source while the influence zone significantly increases. It clearly shows higher thermal diffusivity can facilitate the heat flow in the media.

\section{Conclusion}

In this study an analytical solution to predict soil and moisture content during heat and mass flow in the vicinity of a heat source (e.g., energy pile) is presented. Analytical solutions are necessary to have a better understanding of the physical processes. In addition, they provide convenient tools for numerical model validations and to better understand a problem for engineering projects. In this paper Green's function and the method of separation of variables are employed to investigate the effects of energy pile and soil parameters on transient heat transfer in unsaturated soils. Results indicate that the shape and location of the pile affected the results significantly. Also, the changes of temperature radially are more than the changes in depth in all locations of the piles.

The authors gratefully acknowledge the financial support by the National Science Foundation under Grant No. CMMI- 1804822

\section{References}

1. Man, Y., Yang, H., Diao, N., Liu, J., \& Fang, Z. (2010). A new model and analytical solutions for borehole and pile ground heat exchangers. International Journal of Heat and Mass Transfer, 53(13-14), 2593-2601.

2. Ghasemi-Fare, O., \& Basu, P. (2019). Coupling heat and buoyant fluid flow for thermal performance assessment of geothermal piles. Computers and Geotechnics, 116, 103211.

3. Hartley, J. G., \& Black, W. Z. (1981). Transient simultaneous heat and mass transfer in moist, unsaturated soils. Journal of Heat Transfer, 103(2), 376-382.

4. Eskilson, P., \& Claesson, J. (1988). Simulation model for thermally interacting heat extraction boreholes. Numerical heat transfer, 13(2), 149165.

5. Zeng, H. Y., Diao, N. R., \& Fang, Z. H. (2002). A finite line-source model for boreholes in geothermal heat exchangers. Heat TransferAsian Research, 31(7), 558-567.

6. Zeng, H., Diao, N., \& Fang, Z. (2003). Heat transfer analysis of boreholes in vertical ground heat exchangers. International Journal of Heat and Mass Transfer, 46(23), 4467-4481.

7. Lamarche, L., \& Beauchamp, B. (2007). New solutions for the short-time analysis of geothermal vertical boreholes. International Journal of Heat and Mass Transfer, 50(7-8), 1408-1419.

8. Ghasemi-Fare, O., \& Basu, P. (2013). A practical heat transfer model for geothermal piles. Energy and Buildings, 66, 470-479.

9. Ghasemi-Fare, O., \& Basu, P. (2013). An Annular Cylinder Source Model for Heat Transfer through Energy Piles, Compendium of papers of the 92nd Transportation Research Board (TRB) Annual Meeting, Washington, D.C., January 1317. (No. 13-4949).

10. Ghasemi-Fare, O., \& Basu, P. (2016). Predictive assessment of heat exchange performance of geothermal piles. Renewable energy, 86, 11781196.

11. Cherati, D. Y., \& Ghasemi-Fare, O. (2019). Analyzing transient heat and moisture transport surrounding a heat source in unsaturated porous media using the Green's function. Geothermics, 81, 224-234. 